\title{
Ontology-based Instance Matching for Geospatial Urban Data Integration
}

\author{
Vivek R. Shivaprabhu, Booma Sowkarthiga Balasubramani, Isabel F. Cruz \\ University of Illinois at Chicago \\ \{vrevan2,bbalas3,ifcruz\}@uic.edu
}

\begin{abstract}
To run a smart city, data is collected from disparate sources such as IoT devices, social media, private and public organizations, and government agencies. In the US, the City of Chicago has been a pioneer in the collection of data and in the development of a framework, called OpenGrid, to curate and analyze the collected data. OpenGrid is a geospatial situational awareness platform that allows policy makers, service providers, and the general public to explore city data and to perform advanced data analytics to enable planning of services, prediction of events and patterns, and identification of incidents across the city. This paper presents the instance matching module of GIVA, a Geospatial data Integration, Visualization, and Analytics platform, as applied to the integration of information related to businesses, which is spread across several datasets. In particular, we describe the integration of two datasets, Business Licenses and Food Inspections, so as to enable predictive analytics to determine which food establishments the city should inspect first. The paper describes semantic web-based instance matching mechanisms to compare the Business Names and Address fields.
\end{abstract}

\section{CCS Concepts}

- Information systems $\rightarrow$ Entity resolution; Ontologies; Spatialtemporal systems;

\section{Keywords}

Instance Matching, Record Linkage, Data Integration, Geospatial Data, Ontology

\section{Introduction}

The explosion of digital information, also known as Big Data, includes data collected from several sources such as sensors (environmental, IoT and traditional/mechanical), messages on social media (e.g., Twitter, Facebook), public (city, state, and federal) information, and user reviews (about products and places). Many US cities are working on urban development visions to integrate information and communication technology and Internet of Things to manage their assets in an open but secure fashion. Moving towards the vision of "smart cities" [23], cities are actively creating frameworks that allow the city administrators as well as the general public to explore such data and run queries to identify and monitor incidents across the city, thus creating environments of open and user-driven innovation for experimenting and validating future internet enabled

Permission to make digital or hard copies of part or all of this work for personal or classroom use is granted without fee provided that copies are not made or distributed for profit or commercial advantage and that copies bear this notice and the full citation on the first page. Copyrights for third-party components of this work must be honored For all other uses, contact the owner/author(s).

UrbanGIS'17, November 7-10, 2017, Redondo Beach, CA, USA

(c) 2017 Copyright held by the owner/author(s).

ACM ISBN 978-1-4503-5495-0/17/11.

https://doi.org/10.1145/3152178.3152186 services. By allowing citizens to access and derive conclusions from data, cities are able to promote civic engagement, create developer communities and standardization of tools and techniques, and encourage the process of identification of community needs derived from data [18]. The vision for next-generation urban development is to manage cities' assets, including community services, information systems, infrastructure and businesses, by integrating data collected from heterogeneous sources.

The City of Chicago has been among the top smart cities in the world using information and communication technology that enables the policy makers to communicate directly with the public and analyze city infrastructure to understand how the community is evolving and to be able to provide a better quality of life [23]. By collecting and analyzing data from numerous sources, it is possible to create detailed, informative profiles of the various actors that participate in bringing out a large variety of urban components.

In addition to data portals (like feedingamerica.org), there is now an influx of resources that aggregate data (such as opendatasoft.com) or integrate data from multiple portals/datasets (such as data.gov). Some of these resources link records from multiple sources using known identifiers. The City of Chicago has its own open-source initiative called OpenGrid ${ }^{1}$ that allows citizens to explore their neighborhoods and to enable real-time situational awareness through a map-based user interface [14]. OpenGrid connects to a variety of data sources, including plenar.io [6], which can load multiple datasets from sources such as $\mathrm{CKAN}^{2}$ or Socrata. ${ }^{3}$

In this paper, we present the instance matching module of GIVA, an ontology-based data integration framework, which provides a unified view of data from multiple heterogeneous data sources. We present the instance matching methods as applied to information about businesses in Chicago such as the business name, address, license information, details of inspections, contracts, user reviews, and ratings. The primary difficulties in the integration of such data from disparate sources such as Business Licenses, Food Inspection, Google Places, and Yelp, are mainly due to the different spatial resolutions of data in each dataset, heterogeneities in structure, semantics and modeling, and differences in the data format $[15,19]$. Several domain related challenges are also involved, including (but not limited to) different names for a business such as the Doing Business As (DBA) name and differences in the business names (e.g.: Seven Eleven and 7-11); different addresses representing multiple entrances to the same building; data due to multiple businesses associated with the same entity, and co-located businesses.

GIVA can be coupled with data exploration platforms such as City of Chicago's OpenGrid to integrate the city's geospatial datasets in four modules that involve: (i) data extraction; (ii) instance matching; (iii) a database containing the instance that are matched; and (iv)

\footnotetext{
${ }^{1} \mathrm{http}: / /$ opengrid.io/

${ }^{2}$ http://ckan.org/

${ }^{3}$ http://socrata.com/
} 
a web service API. We explain the framework using two real-time open datasets from the City of Chicago, namely Business Licenses and Food Inspections, and also a subset of the data from Google Places. This framework could easily be extended to include new data sources in the future. The novelty of our approach is its ability to work with multiple datasets (from multiple sources) even if there is no unique key to identify records that belong to a given business (real world entity). Alternate keys such as Business Names and Addresses are often not completely accurate due to human errors/discrepancies during data capture or issues with data integration. A vector of similarity measures are generated using multiple instance matching techniques, which are used to identify records as belonging to the same real world entity and to build consolidated views of each business. Eight datasets from the City of Chicago's data portal $^{4}$ were considered and are listed in Table 1. Implementations are built for two datasets-Business Licenses and Food Inspections. A service with a set of methods to retrieve the consolidated business information for a given business by name is also implemented.

The rest of this paper is organized as follows: Section 2 briefs the importance of geospatial data integration in urban settings, how it is being used, and the rationale behind trying to combine records from disparate sources. Section 3 highlights some of the predominant challenges in integrating geospatial data. Section 4 focuses on our framework for integrating business data of the City of Chicago and details how our framework addresses the challenges mentioned in Section 3, and details the interfaces to extract data from non-uniform source formats, how records are compared using non-Primary Key features such as BusinessNames and Addresses, the data store to house source data as well as the alignment (matching results) and the service to extract the UnifiedBusinessModels for consumption by downstream systems. In Section 5, we discuss the experiment settings and the results of our evaluation using Business Licenses and Food Inspections datasets. In Section 6, we explore related work in geospatial data integration for urban data and the challenges we address using our method. Finally, in Section 7, we offer final remarks and conclude with a discussion of future work. The source code for the application is available online. ${ }^{5}$

\section{Motivation}

Businesses serve as an indicator of economic development of a city. As such, their very existence generates a plethora of data from a variety of sources. This includes:

- Public data such as Business License information for multiple types of licenses (such as Food, Liquor, and Child Services), which have to be renewed annually, Food Inspection records that are generated when a restaurant (or other food establishment) is inspected, various Contracts that are issued by the city for public works or other services, and so on.

- Commercial data including business information such as Addresses and Operating Hours, classification of restaurants by multiple criteria, and other metadata often published in multiple sites such as Yelp, TripAdvisor and Google Places.

- User generated content that may yield structured information (e.g. if a restaurant is kid/family friendly, whether the

\footnotetext{
${ }^{4}$ https://data.cityofchicago.org/

${ }^{5}$ https://gitlab.com/advis/giva
}

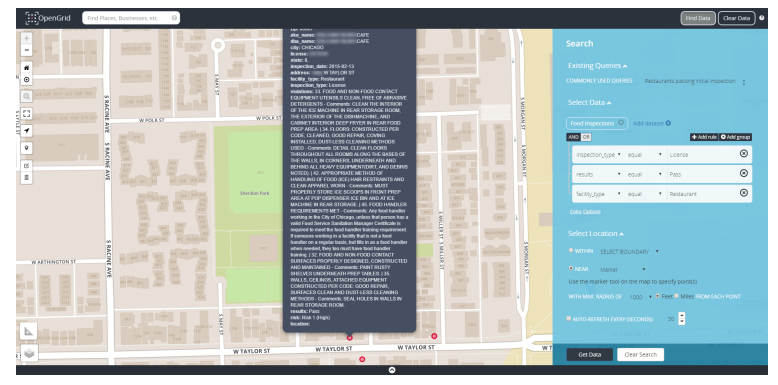

Figure 1: OpenGrid user interface showing food violations for a restaurant near UIC, Chicago.

business is open late) and unstructured data (such as user reviews). This data may be published (possibly differently) across multiple websites and data feeds.

City workers are tasked with researching restaurants and other businesses to gain insights about their operation in order to schedule food inspections, estimate water and electricity usage, design police beat paths, and so on. They have to access multiple datasets, including those that are internal to the city as well as those that are publicly available from third party sources such as Yelp. Existing methods use datasets in a way that does not need them to be combined. Researchers use geospatial features to identify trends in independent datasets and combine summary findings for forecasting. The City of Chicago has successfully used this mechanism to identify food establishments that are most likely to have critical violations so that they may be inspected first.As a result, food establishments with critical violations are more likely to be discovered earlier by inspectors, which results in better overall public health.

Figure 1 shows a screenshot from OpenGrid with food violations for a restaurant in Chicago. Though OpenGrid allows users to search for entities and obtain relevant information from one dataset at a time, it currently does not support a combined view of information from multiple disparate datasets because the datasets do not share common identifiers that uniquely correspond to each real world entity (e.g., a business).

In one of our previous works [2], we describe a method to allow city administrators to use Association Rule Mining over open data to generate patterns, use them for decision making, and better direct limited government resources. By combining features from multiple sources, domain ontologies used in mining trends can be enriched to enable more accurate predictive analytics of urban data.

Combining data from myriad sources allows the creation of a unified view of each business. This provides additional information about individual businesses that can be used for more refined data analysis. In addition to city employees, citizen researchers and students benefit by accessing a unified view of each business. Various organizations, including open source communities and proprietary firms are working independently or in collaboration with the City of Chicago to explore the plethora of datasets such as crime rates, weather patterns, and restaurant reviews. Some of the key use cases we have identified are as follows:

- Eliminate confusion. When users search for records from multiple sources within a small radius of a geo-location, existing systems return hundreds of records that may correspond to 
a handful of businesses in that area. However, there is no straightforward way to discern this without looking at all the records individually. This problem is worsened when the results are plotted on a map, where users will see many points in the same area without knowing if they are multiple businesses in the same building, or records that identify the same business. By creating a unified view of businesses from multiple sources, users are treated with a much smaller result set. This enables them to concentrate on the task at hand instead of trying to de-duplicate records that belong to few businesses.

- Advanced queries. Each business may contain tens to hundreds of records from Business Licenses, Food Inspections and other data sets that may be accumulated together to support advanced queries such as age of the business, total number of active licenses, overall health violations, and so on that can be used to fine tune predictive analytical capabilities available to city workers and researchers alike.

- Break down information silos. By integrating multiple sources, the unified business view will be enriched by features that are specific to each source. For example, the Yelp dataset may present features such as Good for groups. In addition, features such as Overall Rating and User Reviews may be combined to obtain a larger, richer data pool that may eliminate the biases that are inherent about each source.

- Verify accuracy. Features that are common across data sets, such as Legal Name and Operating Hours, can be compared across multiple sources and verified for accuracy. For example, if two sources report one set of Hours and a third source reports a different set of Hours, researchers may choose to trust the first two sources for this feature.

\section{Challenges}

Though various ontology-based data integration frameworks [4, $17,26,28]$ exist, none of them can handle all the challenges in the geospatial domain, and the integration becomes complex to nearly impossible when a new dataset is to be added or an existing dataset is to be modified. We have listed some of the challenges that are quite common in geospatial data integration involving multiple datasets.

(1) Data heterogeneity. Heterogeneity is typically caused by various factors such as autonomous data, differences in data sources, disciplines, tools, events, data models, data collection and storage techniques, and so on. Data heterogeneity encompasses several categories such as syntactic heterogeneity, structural or schematic heterogeneity, semantic heterogeneity, and heterogeneities in conceptual and spatial modeling.

(2) Inconsistency. It is caused mainly due to the imperfection in data, thereby affecting the data quality. For example: two different values for the same attribute in two datasets.

(3) Update frequency. It is the frequency in which the data is modified, updated, or released. For example, weather and traffic data are available hourly, whereas the administrative boundaries are available on an annual cycle. The update frequency influences the tracking of events.

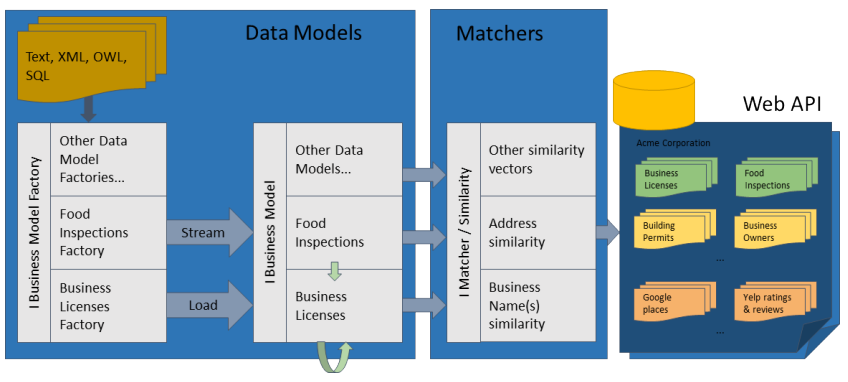

Figure 2: System architecture.

(4) Data instability. Stability refers to the steadiness of format, schema and other aspects of data. Instability in data may lead to invalid outcomes in the integrated data. Continuous access to data is one feasible solution to data instability.

Narrowing the aforementioned issues with geospatial data integration to the business datasets of the City of Chicago, poses to the following challenges:

(1) Heterogeneous data. Data sources provide data extracts in a variety of data formats (such as CSVs, OWLs or database extracts) containing values that are not standardized or represented using varying spatial resolutions and semantics $[15,19]$. Every system uses different identifiers and are not guaranteed to be consistent across sources.

(2) Data quality. Values entered manually are prone to typographical errors and representational variations (Example: 7-Eleven vs. 7-11) that make it difficult to use attribute data directly in the matching process.

(3) Multiple names and addresses. Businesses can have multiple names such as Doing-Business-As (DBA) name, Legal name and Also-Known-As (AKA) name; buildings can have multiple addresses associated with multiple entrances; many businesses typically coexist within the same building.

(4) Multiple records per real world entity. Each business will have multiple records in most sources. For example, multiple types of licenses issued annually to each business; restaurants undergoing several food inspections; and so on.

(5) Increase in the number of sources. Although current work is focused on two sources, the city of Chicago intends to expand the framework to integrate multiple internal (such as Contracts and Permits) and external (such as Google Places and Yelp) datasets in the future.

\section{Framework}

This section describes how the GIVA framework addresses the challenges discussed in Section 3. The GIVA system architecture is shown in Figure 2. The goal is to combine various aspects of businesses from multiple sources into a unified data model using an array of geospatial instance matching techniques. The design allows for source data containing information about businesses to be supplied in a variety of file formats (such as CSV, OWL or XML). An interface defines the methods that can be implemented to add new data sources and file formats, which will extract the necessary fields-primarily Business Names and Addresses-into a common class/structure. Once the data model is normalized, records from the source are compared with the existing records from an underlying 


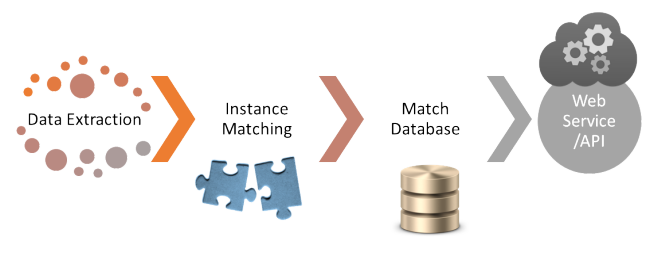

Figure 3: Phased approach for instance matching.

database. This database, which is empty at first, contains unified data model instances of previously processed businesses.

An array of techniques determine similarity between pairs of records based on several criteria that do not require values in all fields to be exactly equal (such as ISub similarity and weighted Jaccard measure); the results are combined into a single similarity value for each pair. If multiple Business Names and/or Addresses exist, all values are compared and an average score is determined. Blocking [1] is used to avoid combinatorial explosion using known fields that have to be exactly the same between pairs of records being compared (like City, State and Zip Code). Instances that have a combined similarity value higher than a preset threshold are identified (known as a match) as belonging to the same business (real world entity), merged into a unified data model and stored in the database. Thus, an ontology of unified business instances that represents the real world entities is built where the source records are linked as child entities that represent various aspects of the businesses. If all similarity values are below the threshold (non-match), a new instance that represents a new business is created in the database. The process is repeated until all records from all sources are processed. Instance Matching is a time consuming process, and hence, is performed in an offline/batch mode.

The results of the instance matching process that are stored in the database (also known as an Alignment) are served to end users through a service that takes Name as a parameter and returns all unified business model instances that contain the query parameter in their Business Names. This framework is able to address all of the challenges identified as part of the geospatial data integration problem. Several benchmarks to evaluate the efficacy of the solution are considered $[5,10]$. The implementation is divided into four phases as shown in Figure 3.

\subsection{Data Extraction}

In order to compare records from multiple sources, the relevant (non-key) fields that are used to identify each business-Business Names and Addresses-are extracted from each dataset. Each new data source should contain sufficient information both in terms of available attributes as well in terms of the number of records. For example, a data set that just lists the names and addresses of businesses in Chicago will not add any value to the consolidated record. Similarly, a dataset that contains 100 records may not provide enough magnitude to be considered worthwhile. A critical dataset that adds significant value (for example, a list of debarred businesses) should be considered even if it adds only one additional attribute of data for a small number of businesses. Business Licenses and Food Inspections datasets seem to have sufficient breadth of features as well as a large enough data size, as shown in Table 1.

A data model interface is built to ensure that any source that is used in instance matching, at a minimum, guarantees the existence

\begin{tabular}{l|c|l}
\hline Dataset & Decision & Reason \\
\hline Business Licenses & Use & $>876 \mathrm{k}$ records \\
Food Inspections & Use & $>335 \mathrm{k}$ records \\
Building Permits & Cannot use & No business name \\
Payments & Cannot use & No address \\
Business Owners & Cannot use & No address \\
Condom Distribution Sites & Cannot use & Only 178 records \\
Debarred Firms/Individuals & Cannot use & Only 153 records \\
Grocery Stores & Cannot use & Only 507 records \\
\hline
\end{tabular}

Table 1: Datasets considered from the data portal.

of the features required for matching-Source Name and ID, Business Names and Address components. The application is designed to be sufficiently modular to enable integration of new sources with little effort. This interface is implemented for Business Licenses and Food Inspections. There is sufficient separation of concerns to guarantee that data for the source can be provided in any of the supported file formats (such as CSV and OWL).

A second interface is built to convert data from an arbitrary file format (such as CSV, XML and OWL) into a feature set that can be handled as a standard object type in downstream processes. The interface guarantees that any implementation will provide methods to take a record and return an array of features or a ResultSet and a method that specifies if a given record should be processed or not (this is useful when the dataset needs to be filtered). An implementation of this interface provides the constructs necessary to read CSV files given the list of fields to expect in the file, not necessarily in the order provided. So, if the expected fields are A, B, and C, and the input file contains $\mathrm{B}, \mathrm{C}, \mathrm{A}$, and $\mathrm{D}$, the reader is intelligent to get the necessary data based on the header fields in first line, and ignore the additional column from the file. Also, quoted string values that may include commas are handled appropriately. Two classes based on the abstract factory are defined-for Business Licenses and Food Inspections. The result of this step is a standardized set of IBusinessModel instances, which can be used for Instance Matching. No other file formats are implemented since both sources are available as CSV. Implementation of other file formats is straightforward for when another file format is chosen for datasets that will be integrated. If the data is from a nested data source such as XML or JSON, the schema may have to be flattened first.

\subsection{Alignment}

Ideally, each real world entity (in this case: business + location) will have a unique identifier that is used by all sources, both internal (to the city) and external (third party), that can be used to identify the records that belong to the same real world entity. For US citizens, this could be the Social Security Number; for businesses, this could be a location-specific Business License number; and so on. However, none of the datasets share such an identifier, which can be reliably used to perform joins.

Two non-key feature-sets-Business Names and Address components-are used to perform Instance Matching. Input data implements the IBusinessModel interface which guarantees that every record has these features. The process begins with an empty set of UnifiedBusinessModels. Every record from the input is compared with the existing set of UnifiedBusinessModels looking for an equivalent IBusinessModel. If it is found, the record is added to its collection. 


\begin{tabular}{|c|l|l|c|}
\hline$\#$ & Food Inspection & Business License & Score \\
\hline 1 & New City Daycare Inc., 1000 W 51st St \#1, 60609 & New City Daycare Inc., 1000 W 51st St \#1, 60609 & $\mathbf{1}$ \\
\hline 2 & Vireva Nursey School, 1935 W 51st St, 60609 & Vireva Nursery School, 1935 W 51st St, 60609 & $\mathbf{0 . 9 8}$ \\
\hline 3 & Smart Start Development Center, 7201 S Western Ave, 60636 & Smart Start Development Center, Inc., 7201 S Western Ave, 60636 & $\mathbf{0 . 9 4}$ \\
\hline 4 & Jellybean Learning Center, 7411 S Cottage Grove Ave, 60619 & Jellybean Learning Center, 358-370 E 71st St, 60619 & 0.53 \\
\hline 5 & City Garden Early Childhood Center, Inc., 920 W 19th St, 60608 & Early Childhood Educare Center, 5044 S Wabash Ave, 60615 & block \\
\hline
\end{tabular}

Table 2: A sample set of matching results showing the various types of differences (emphasized) between source and target datasets. The last column shows the average value of the similarity scores computed each Business Name and Address fields.

If not, a new UnifiedBusinessModel is created. This process is repeated until all data from all sources are processed. The result of the matching process is a set of UnifiedBusinessModels, each of which contains a unique identifier (UUID), a set of IBusinessModels that groups records that belong to the same real world entity.

Ontologies. An ontology is a formal, explicit specification of a shared conceptualization $[11,16]$. It provides a common vocabulary to model the schema for a system as well as represent entities from the domain. Ontologies are typically stored using RDF or OWL formats, but may also be represented using attributes in flat files (such as CSV) when unique parent-child relationships are represented in each instance. Two source (spatial and business) ontologies and one target ontology are built, as follows:

- A spatial ontology organizes records into a hierarchical structure based on Country, State, City, and Zip Code (in that order), as shown in Figure 4. Although we know that all businesses in this exercise will be from Chicago, the solution is designed to be expandable to more than one city. By classifying data instances into this hierarchy, we can minimize the combinatorial explosion of comparisons by limiting potential pairs to belong to the same leaf node of the tree structure.

- A business ontology classifies establishments based on Type of Business, such as Restaurants, Children's Services, and Motor Vehicle Services, which are further classified into SubTypes. Using these classifications, two businesses with similar names across Business Types are not compared, thus saving time as well as improving the quality of the results.

- The target ontology consists of instances of the unified business model. Each instance will contain a hierarchical structure of data objects that represent various aspects of the business, including various Business Licenses, Food Inspections, Operating Hours, User Ratings, Reviews, and other metadata.

AgreementMakerLight (AML).. This ontology matching system is a collaborative effort between LASIGE at the University of Lisbon and the ADVIS Lab at UIC, a successor of the AgreementMaker system for matching efficiently large real-world schemas and ontologies [7]. AML is a lightweight system that is designed to match very large ontologies efficiently for a wide variety of domains [13] AML was the top performing system in five tracks and one of the top performing systems in three others at OAEI in 2016 [12]. Our implementation reuses some methods from AML, along with others that are built from scratch, to build a vector of similarity measures for each pair of records compared.

Similarity Methods. An ISimilarity interface allows the creation of custom matchers that can be used to identify the similarity for a

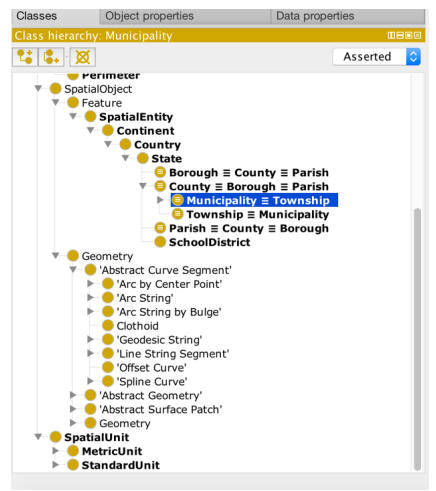

Figure 4: Spatial ontology representation, stored as a CSV.

feature. An implementation of this interface is created to compare BusinessNames and reused to compare Addresses. Depending on the features in future datasets, implementation of new custom matchers is straightforward. The BusinessNameSimilarity matcher uses the following techniques to find the similarity between two strings. Each similarity measure is assigned a weight (based on a heuristic analysis of the data) and the maximum value of all the similarities is used as the final similarity measure. The following methods are used:

(1) string.equals: If two strings are exactly equal, no further processing is required.

(2) aml.ISub.similarity: AML implements ISub similarity [24]; it is similar to the Levenshtein distance, based on the length of common substrings; and it returns similarity between names with high accuracy.

(3) cleanISub: ISub similarity on cleaned variants (by removing all special characters) from input strings.

(4) aml.weightedfaccard: AML's implementation of Weighted Jaccard similarity; it measures the overlap between two strings with their component word parts.

Techniques to remove stop words (such as 'the') and to identify acronyms of business names (for example, 'KFC') were developed, but disabled because they were generating too many false positives.

Blocking [1] is the principle of controlling combinatorial explosion at the onset by using known variables to filter out pairs of records that do not have matching values. For a given pair of records, the values for City, State and Zip Code are compared using string.equals. When available, a similar comparison is applied for Business Types. If they are not equal, the pair is discarded as nonmatching. This process restricts comparisons to records for which the particularly discriminating identifiers agree.

Each IBusinessModel will return one or more values for the Names feature. Every combination of this feature from both records are 


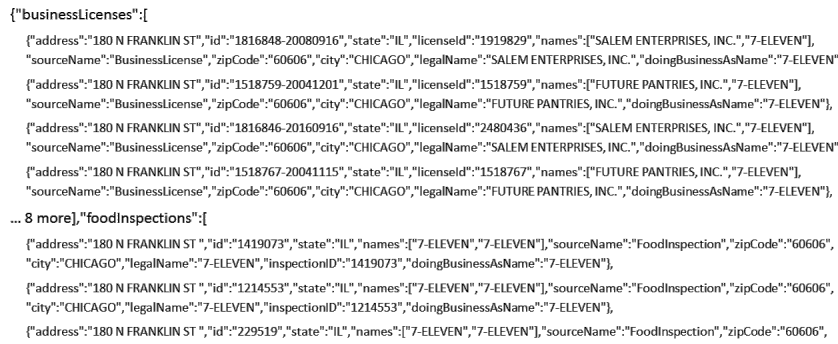

Figure 5: Sample JSON response.

compared to check if their overall similarity is higher than the required threshold. Determining where to set the matching/nonmatching thresholds is a balancing act between obtaining an acceptable sensitivity and positive predictive value. During evaluation in collaboration with the City of Chicago, we evaluated the quality of hundreds of matching results for various test values of thresholds for both Business Names and Address similarity matching processes. Using this heuristic method the following threshold values were selected: A very high threshold (90\%) is used to obtain business names that are very similar. This reduces recall, but it has been determined that high precision is more important for this situation. Street Address from both records are compared with a very high threshold (95\%) to confirm that the business is at the same location.

If both similarity measures are higher than the threshold, then the source (the incoming/new record from the input) is merged into the existing UnifiedBusinessModels). If not, a new UnifiedBusinessModel is created for the source record, which will be used in comparison of subsequent input records.

Data store. The source datasets are stored as-is in corresponding tables of a relational database. This data is not used in the matching process, but may be used to provide additional information to users of the service/API. The results of the matching process are stored in a source-agnostic table, that stores the features exposed by the IBusinessModel along with the globally unique identifier assigned to the UnifiedBusinessModel. The SOURCE and SOURCE_ID columns in the BusinessModelEntity table, which stores the alignments, are used to refer to the records identified by the Primary Keys of the source tables. This Foreign Key relationship constraint is not enforced in the database, but is critical to identify the records in the source datasets because the BusinessModelEntity table only stores values for attributes used in matching; the remaining features that add value to the consolidated view are joined from the source tables into the response object that is returned by the service/API, as described in Section 4.3.

\subsection{Service/API}

The City intends to make the results of the matching process open and available to anyone who wish to access it. The match results are intended to be served to downstream systems, including OpenGrid, Plenario, and any other system that the community of developers may choose to use it for. A RESTful Web API is designed, that takes in Business Name and/or Address as query parameters and returns a JSON or XML document containing the UnifiedBusinessModels that satisfy the parameters.
A sample of the match results are shown in Figure 5. Each UnifiedBusinessModel consists of all the records from the various sources that belong to the same real world business entity, grouped by source. Thus, instead of seeing tens (if not hundreds) of results for a given location that may contain multiple results for each business, the resultset contains a handful of records that represent unique businesses; all details that represent the history of each business is returned as an ontology containing various aspects of the business, such as Business Licenses, Food Inspections, and so on.

A service that returns the requested data has been implemented. Development of a Java component that will provide results to web queries from a RESTful API is part of the future goal.

\section{Experimental Evaluation}

Instance Matching Mode. This process takes two (or more) datasets as parameters (in this case, Business Licenses and Food Inspections). Input parameters, connection strings, file locations, etc are provided in a .properties file. First, the Alignment Database is truncated; this can be turned off to enable incremental inserts. Records extracted from the datasets (in this case, CSV files) are filtered based on filter conditions (such as Zip code) and transformed into IBusinessModel objects. Similarity vectors are generated for each incoming record paired with the Alignment Database. Blocking ensures that only relevant pairs of records are compared. If there is a match, the existing UnifiedBusinessModel is updated. If not, a new UnifiedBusinessModel is created. The results are stored in the Alignment Database.

Table 2 shows a sample set of records that are compared using GIVA. The first pair is exactly equal, hence a similarity score of 1. Lines 2 and 3 show that the addresses are the same, but the business names are slightly different; they are identified as the same business since the overall similarity score is higher than the selected threshold (0.90). Line 4 shows a pair of records for the same business at different locations; its score is much lower than the threshold and identified as non-matching. The last pair is blocked from instance matching since they have different zip codes.

The instance matching process was executed on data from a single zip code-60606. The nature of the solution implies having to compare every record in the source with every record in the target dataset. Blocking is implemented to prevent combinatorial explosion using City, State, and Zip Code. This reduces the number of comparisons and eliminates pairs of records which are definitely unequal. The city creates multiple business licenses for each business (one for each year of its existence). From the 16,853 business licenses processed, 3,522 unique business entities were created by matching the Business License dataset with itself. 2,179 food inspections were processed for this zip code; 2,108 (96.7\%) were matched to a business entity (as created from the previous step). 71 food inspection records did not have matching businesses, most of which were because inspectors did not record a valid address, or since blocking eliminated the restaurant as its zip code from the Business License and the Food Inspection records were not equal.

A reference alignment was built using data from the candidate keys, such as License Number. This was possible because both source and target datasets were obtained from the city's data portal. Sample data from the instance matching results are verified by working in collaboration with the Advanced Analytics team at the city, and found to be accurate. 


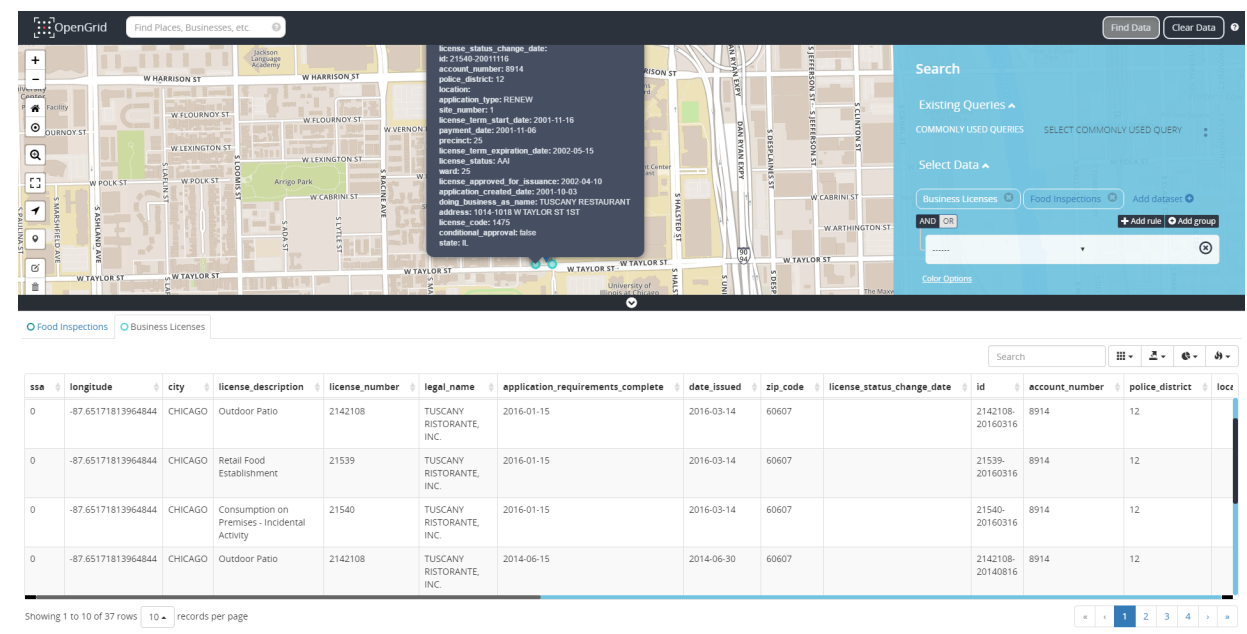

Figure 6: OpenGrid user interface shows 37 Business Licenses and 10 Food Inspections for a restaurant in Chicago.

Service Mode. In this mode, the application takes a (partial) business name as a parameter and returns UnifiedBusinessModels from the Alignment Database whose Names are equal to or contains the requested parameter. This is a set of functions that will service web requests, designed to be fast, to be called from the RESTful API. The results are returned as a JSON document, which can be consumed by applications such as OpenGrid.

Users can select one or more datasets to view in a map-based interface in OpenGrid. They can apply various filters based on fields in the datasets and/or use a spatial marker and radius to look at data for a locality. Records that satisfy the criteria are displayed in the form of pins on a map view. They can then click on a pin to obtain detailed information (such as issued licenses and food violations) from the selected datasets.

Figure 6 shows the result of a sample geospatial search (using a marker and $100 \mathrm{ft}$. radius). The list view in the lower half shows results from Business Licenses (37 records) and Food Inspections (10 records) datasets viewed as two tabs. Detailed information about the selected business is displayed as a pop-up. Using this graphical map-based interface, users can look at the various types of licenses issued to the restaurant and obtain detailed information about food inspections statuses and violations recorded by inspectors.

\section{Related Work}

In the previous sections, we have highlighted several issues with respect to geospatial data integration. Though various ontologybased data integration frameworks exist, none of them could handle all the challenges in the geospatial domain, and the integration becomes complex to nearly impossible when a new dataset is to be added or an existing dataset is to be modified.

Hong et al. [17] propose a solution for semantic heterogeneity on cross-domain applications. They use lightweight ontologies for integration, by considering common predicates among the data sources. However, differences in the modeling perspective, scope difference, and different granularities of the domains cause some issues in detecting the common predicates. Also, this approach is unsuitable to handle some cases, such as when the number of concepts in one dataset is much larger than in the other dataset.

Tran et al. [26] handle spatial data from various sources by resolving structural and semantic heterogeneity. This model is very expressive, and uses reasoners to allow cross-database queries with reasoning on the spatial, temporal, as well as the spatio-temporal relationships. However, their methodology focuses on land-use datasets and cannot be applied to other domains because their matching algorithm relies on a land-use specific ontology.

Stoimenov et al. [25] propose a semantic interoperability approach, but is only convenient when integrating two datasets. It is not straightforward to consider more than two datasets. In addition, only limited semantic relationships are considered. For example, multiple spatio-temporal resolution is not considered.

Beck et al. [4] propose the integration of datasets in the utilities domain. They take into account various forms of heterogeneities, but they require major changes when a new dataset is to be integrated, one of the reasons being that they consider a single ontology, thus making it difficult to integrate datasets with different views on a domain, whose representation would need multiple ontologies.

Mai et al. [21] introduce a 'follow-your-nose' style of visual interface when alignments already exist between two (or more) ontologies. They propose several methods that allows users to navigate between entities from various large (in their example, $45 \mathrm{M}$ triples) geodata sources. However, this method does not combine multiple sources based on similarity features and assumes that instances in different datasets represent distinct real world objects.

Introducing human in the loop allows users to validate matching results and enables a system to automatically modify parameters (such as similarity thresholds) that are used in the matching process $[3,8]$. Together with the visualization process, analytical methods form the backbone of a visual analytics approach to ontology matching $[9,20]$.

SocialGlass is a framework proposed by Psyllidis et al. [22], which is aimed at the visual exploration of large-scale and heterogeneous urban data. Among other data sources, this system relies mostly on crowdsourcing (e.g., data from social media such as 
Facebook and Twitter) to collect data related to events. Our system differs from SocialGlass in a way that it is able to handle data not just related to events, but also traditional datasets such as Business Licenses, Contracts, and so on.

We are also currently working on performing predictive analytics using text classification for restaurant inspections based on Yelp reviews. This approach extracts features from existing Yelp reviews and uses various machine learning algorithms such as SVM, RNN, Naive Bayes, and Random Forest to identify restaurants that require immediate inspections [27].

\section{Conclusions and Future Work}

Integration of multiple sources of information will help expand the depth of information that can be provided for each business in an urban setting. In this demo paper, an ontology-based framework is introduced that enables instance matching in the City of Chicago's OpenGrid web platform to explore, integrate, and analyze geospatial data. Third party sources will not have values for candidate keys, or they will use their own version of the key which will not map to keys from other sources. Therefore, when external sources are used, validating the results of the alignment may prove to be difficult. A web service will enable the easy retrieval of UnifiedBusinessModels, which may be consumed by end users as well as downstream systems that the city intends to integrate these data with. Like the OpenGrid code, the code of the work described in this paper has been open-sourced for independent software developers to contribute to and/or provide feedback.

Future work will focus on the extraction of business information from Google Places and Yelp to be consolidated with existing business entities. The key challenge of using external sources lies in validating the results of the alignment. This is because, typically, third party sources lack values for candidate keys, or they use their own version of the key that do not map to the keys from other sources. Once the RESTful web API is exposed, OpenGrid will be updated to consume the results of the matching exercise to visualize data from multiple sources that belong to the same business as a single record. Extension of this framework to accommodate other applications such as surveillance for malaria elimination based on health care facilities, Yelp reviews, tweets, and land use is also part of our future plans.

\section{Acknowledgments}

We thank Tom Schenk, Chief Data Officer of the City of Chicago, and his team at the Department of Innovation \& Technology, especially Gene Leynes. This work was partially supported by NSF awards CNS-1646395, III-1618126, CCF-1331800, and III-1213013, by a Bill \& Melinda Gates Foundation Grand Challenges Explorations grant, and by the Bloomberg Philanthropies Mayor's Challenge Award Chicago SmartData Platform. Supplementing the permissions stated in the first page, this work is licensed under a Creative Commons Attribution 4.0 International License (http://creativecommons.org/ licenses/by/4.0/).

\section{References}

[1] Aizawa, A., And Oyama, K. A Fast Linkage Detection Scheme for Multi-source Information Integration. In International Workshop on Challenges in Web Information Retrieval and Integration (2005), pp. 30-39.
[2] Balasubramani, B. S., Shivaprabhu, V. R., Krishnamurthy, S., Cruz, I. F., AND MALIK, T. Ontology-based Urban Data Exploration. In ACM SIGSPATIAL Workshop on Smart Cities and Urban Analytics (UrbanGIS) (2016), pp. 10:1-10:8.

[3] Balasubramani, B. S., Taheri, A., ANd Cruz, I. F. User Involvement in Ontology Matching Using an Online Active Learning Approach. In ISWC International Workshop on Ontology Matching (OM) (2015), vol. 1545 of CEUR-WS, pp. 45-49.

[4] Beck, A., Fu, G., Cohn, A., Bennett, B., And Stell, J. A Framework for Utility Data Integration in the UK. In UDMS Symposium (2007), pp. 261-276.

[5] Castano, S., Ferrara, A., Montanelli, S., and Varese, G. Ontology and Instance Matching. In Knowledge-driven multimedia information extraction and ontology evolution. Springer, 2011, pp. 167-195.

[6] Catlett, C., Malik, T., Goldstein, B., Giuffrida, J., Shao, Y., Panella, A., Eder, D., van Zanten, E., Mitchum, R., Thaler, S., Et Al. Plenario: An Open Data Discovery and Exploration Platform for Urban Science. IEEE Data Eng. Bull. 37, 4 (2014), 27-42.

[7] Cruz, I. F., Palandri Antonelli, F., and Stroe, C. AgreementMaker: Efficient Matching for Large Real-World Schemas and Ontologies. PVLDB 2, 2 (2009), 1586-1589.

[8] Cruz, I. F., Palmonari, M., Loprete, F., Stroe, C., And Taheri, A. Quality-Based Model for Effective and Robust Multi-User Pay-As-You-Go Ontology Matching. Semantic Web 7, 4 (2016).

[9] Cruz, I. F., Stroe, C., and Palmonari, M. Interactive User Feedback in Ontology Matching Using Signature Vectors. In IEEE International Conference on Data Engineering (ICDE) (2012), pp. 1321-1324.

[10] Daskalaki, E., Flouris, G., Fundulaki, I., and Saveta, T. Instance Matching Benchmarks in the Era of Linked Data. Web Semantics: Science, Services and Agents on the World Wide Web 39 (2016), 1-14.

[11] Euzenat, J., ANd Shvaiko, P. Ontology Matching, vol. 18. Springer, 2007.

[12] Faria, D., Pesquita, C., Balasubramani, B. S., Martins, C., Cardoso, J., CuRAdo, H., Couto, F. M., AND Cruz, I. F. OAEI 2016 Results of AML. ISWC International Workshop on Ontology Matching (OM) (2016), 138.

[13] Faria, D., Pesquita, C., Santos, E., Cruz, I. F., And Couto, F. M. AgreementMakerLight: A Scalable Automated Ontology Matching System. Data Integration in the Life Sciences (DILS) (2014), 29.

[14] Fazio, D., Giovannini, E., ANd Signore, M. Data Ecosystems: A New Challenge for Official Statistics. In European Conference on Quality in Official Statistics (2016).

[15] Gotway, C. A., And Young, L. J. Combining Incompatible Spatial Data. fournal of the American Statistical Association 97, 458 (2002), 632-648.

[16] Guarino, N., Oberle, D., And StaAB, S. What Is an Ontology? Springer Berlin Heidelberg, 2009, pp. 1-17.

[17] Hong, J.-H., AND KuO, C.-L. A Semi-automatic Lightweight Ontology Bridging for the Semantic Integration of Cross-domain Geospatial Information. International fournal of Geographical Information Science 29, 12 (2015), 2223-2247.

[18] Kassen, M. A Promising Phenomenon of Open Data: A Case Study of the Chicago Open Data Project. Government Information Quarterly 30, 4 (2013), 508-513.

[19] LE, Y. Challenges in Data Integration for Spatiotemporal Analysis. Fournal of Map \& Geography Libraries 8, 1 (2012), 58-67.

[20] Li, Y., Stroe, C., AND CruZ, I. F. Interactive Visualization of Large Ontology Matching Results. In ISWC International Workshop on Visualizations and User Interfaces for Ontologies and Linked Data (Voila!) (2015), vol. 1456, CEUR-WS, pp. 37-48.

[21] Mai, G., Janowicz, K., Hu, Y., And McKenzie, G. A Linked Data Driven Visual Interface for the Multi-perspective Exploration of Data Across Repositories. In ISWC International Workshop on Visualizations and User Interfaces for Ontologies and Linked Data (Voila!) (2016), pp. 93-101.

[22] Psyllidis, A., Bozzon, A., Bocconi, S., ANd Titos Bolivar, C. A Platform for Urban Analytics and Semantic Data Integration in City Planning. Springer, 2015, pp. 21-36.

[23] Schaffers, H., Komninos, N., Pallot, M., Trousse, B., Nilsson, M., and Oliveira, A. Smart Cities and the Future Internet: Towards Cooperation Frameworks for Open Innovation. In The Future Internet Assembly (2011), Springer, pp. 431-446.

[24] Stoilos, G., Stamou, G., And Kollias, S. A String Metric for Ontology Alignment. In International Semantic Web Conference (2005), Springer, pp. 624-637.

[25] Stoimenov, L., Stanimirovic, A., and Djordjevic-Kajan, S. Semantic Interoperability Using Multiple Ontologies. AGILE (2005), 26-28.

[26] Tran, B.-H., Plumejeaud-Perreau, C., Bouju, A., and Bretagnolle, V. A Semantic Mediator for Handling Heterogeneity of Spatio-temporal Environment Data. In Research Conference on Metadata and Semantics Research (2015), Springer, pp. 381-392.

[27] Wang, Z., Balasubramani, B. S., and Cruz, I. F. Predictive Analytics Using Text Classification for Restaurant Inspections. In ACM SIGSPATIAL Workshop on Smart Cities and Urban Analytics (UrbanGIS) (2017).

[28] Zhang, L., Ma, Y., AND Wang, G. An Extended Hybrid Ontology Approach to Data Integration. In International Conference on Biomedical Engineering and Informatics (BMEI) (2009), IEEE, pp. 1-4. 